\title{
Quantitative Trait Loci for the Diurnal Flag Leaf Starch Content during the Early Grain-filling Stage in Wheat (Triticum aestivum L.)
}

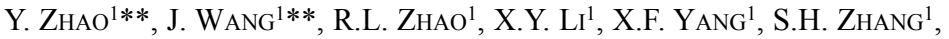 \\ J.C. TIAN ${ }^{2}$ and X.J. YANG ${ }^{1 *}$ \\ ${ }^{1}$ Huabei Key Laboratory of Crop Germplasm, Hebei Agricultural University, \\ Baoding 071000, Hebei, China \\ ${ }^{2}$ Shandong Agricultural University, Tai'an 271018, China
}

(Received 14 April 2017; Accepted 25 July 2017)

\begin{abstract}
Starch is a product of photosynthetic activities in leaves. Wheat yields largely depend on photosynthetic carbon fixation and carbohydrate metabolism in flag leaves. The mapping of quantitative trait loci (QTLs) associated with flag leaf starch content (FLSC) in wheat (Triticum aestivum L.) was completed using unconditional and conditional QTL analyses. The FLSC of this population during the early grain-filling stage was measured at six stages in six environments. Combining unconditional and conditional QTL mapping methods, eight unconditional QTLs and nine conditional QTLs were detected, with five QTLs identified as unconditional and conditional QTLs. Four unconditional QTLs (i.e. $q F L S-1 B, q F L S-1 D-1$, $q F L S-4 A$, and $q F L S-7 D-1$ ) and one conditional QTL (i.e. $q F L S-3 A-1$ ) were identified in two of six environments. Two QTLs ( $q F L S-1 D-2$ and $q F L S-7 D-1)$, which significantly affected the FLSC, were identified using the unconditional QTL mapping method, while three QTLs (i.e. $q F L S-1 A, q F L S-3 A-1$, and $q F L S-7 D-1$ ) were detected using the conditional QTL mapping method. Our findings provide new insights into the genetic mechanism and regulatory network underlying the diurnal FLSC in wheat.
\end{abstract}

Keywords: wheat (Triticum aestivum L.), quantitative trait loci, developmental behaviour, starch

\section{Introduction}

Starch is a product of photosynthetic activities in leaves (Zeeman et al. 2007). A large proportion of the carbon assimilated in leaves during the day is transiently stored in chloroplasts as starch, and is degraded at night (Weise et al. 2004; Ishimaru et al. 2005). The synthesis and degradation of starch need to be well regulated to ensure that the starch temporarily stored in leaves supplies enough carbon and energy for growth and metabolic activities in plants that accumulate starch (Fondy and Geiger 1985; Fondy et al. 1989; Smith et al. 2003; Zeeman et al. 2004). Wheat yields largely depend on photosynthetic carbon fixation and carbohydrate metabolism in flag leaves (Jenner et al. 1991).

*Corresponding author; E-mail: shmyxj@126.com; Phone: +86-0312-7528260; Fax: +86-0312-7528265;

**Both authors contributed equally to this work. 
Under normal conditions, about $30 \%$ of the yield is determined by the accumulated carbohydrate content during the grain-filling stage (Matsushima 1966; Ishimaru et al. 2005).

The development of molecular genetics research techniques has enabled the selection of genes using molecular markers (i.e. marker-assisted selection) during the breeding process (Kato et al. 2000). The starch content in wheat leaves is generally considered to be controlled by quantitative genes (Ishimaru et al. 2007). The pyramiding of positive alleles (expressed at consecutive stages or high phenotypic variation) from different parents by marker-assisted selection may lead to increased flag leaves starch content, thereby increasing the grain-filling intensity and improving wheat yields (Xu and Zhao 1995; Ishimaru et al. 2007). Molecular mapping experiments have revealed that several quantitative trait loci (QTLs) regulate starch contents in plants. Calenge et al. (2006) exploited natural variations in Arabidopsis thaliana to decipher the genetic architecture influencing carbohydrate contents and identified 13 QTLs associated with starch. Ishimaru et al. (2005) identified a new locus ( $r g 5$ ) related to improved carbohydrate storage capacity and maintenance of high sink activity. This locus is responsible for increasing yield potentials in various rice (Oryza sativa L.) genetic backgrounds. Ishimaru et al. (2007) studied the storage of starch in rice plants before heading using QTL analyses of a population of backcross inbred lines. The mapped QTLs explained $42 \%$ of the phenotypic variance on chromosomes 4 (G235) and 5 (C1268). In these QTL studies, leaf starch contents were measured at only one stage without considering the effects of distinct gene expression patterns at different developmental stages.

Conditional and unconditional QTLs for the traits were examined using an improved statistical mapping method (Zhu 1995; Wang et al. 1999). Several recent studies have explored the quantitative traits in wheat ( $\mathrm{Li}$ et al. 2015; Xu et al. 2017; Zhang and Wang 2015). To the best of our knowledge, there are no reports describing the analysis of QTLs for wheat flag leaf starch content (FLSC). In this study, a doubled haploid (DH) population derived from a Huapei $3 \times$ Yumai 57 cross was used to investigate the QTLs associated with temporal changes to the wheat FLSC under six environmental conditions. Conditional and unconditional QTLs for the FLSC were examined using a statistical mapping method. The objectives of this study were to: (1) characterize the expression of QTLs associated with the FLSC using conditional and unconditional QTL mapping methods and (2) clarify the genetic basis of the accumulation of starch in wheat flag leaves. Our findings provide new insights into the genetic mechanism and regulatory network underlying the diurnal FLSC in wheat.

\section{Materials and Methods}

\section{Plant materials}

For linkage map construction, we used a population of $168 \mathrm{DH}$ lines produced by hybridization with maize pollen of wheat $\mathrm{F}_{1}$ hybrid plants from the cross between two Chinese wheat cultivars Huapei 3/Yumai 57, which were, respectively, released by Henan 
Province, China in 2006 (Hai and Kang 2007) and nationally in 2003 (Guo et al. 2004). The two parents, which are cultivated over a large area of the Yellow and Huai valley wheat region of China, differ with respect to several agronomically important traits as well as some related to baking quality (Guo et al. 2004; Hai and Kang 2007; Zhao et al. 2010).

\section{Experimental design}

A total of 168 lines and their parents were grown at the following three sites in China: experimental farm of the Institute of Crop Sciences-Academy of Agricultural Sciences in Xingtai (i.e. $E_{1-1}$ in 2011 and $E_{1-2}$ in 2012), Stock Seed Farm in Cangzhou (i.e. $E_{2-1}$ in 2011 and $E_{2-2}$ in 2012), and the Academy of Agricultural Sciences in Baoding (i.e. $E_{3-1}$ in 2011 and $\mathrm{E}_{3-2}$ in 2012). The experimental design followed a randomized complete block design with three replications. Plants were grown under normal field conditions in threerow plots ( $2 \mathrm{~m}$ long with between-row spacing of $25 \mathrm{~cm}$ ), with 20 seeds sown in each row, interplant was $10 \mathrm{~cm}$ distance. The flowering time of each line was recorded and at least 50 simultaneously flowering spikes in the DH lines were tagged, and collected leaf tissue 10 days after anthesis. Eight leaves tagged spikes from each line were sampled in a 4-hour interval starting from 7:00 up to 3:00 (i.e. 7:00, 11:00, 15:00, 19:00, 23:00, and 3:00, which were designated as $\mathrm{T}_{1}, \mathrm{~T}_{2}, \mathrm{~T}_{3}, \mathrm{~T}_{4}, \mathrm{~T}_{5}$, and $\mathrm{T}_{6}$, respectively) and stored at $-70{ }^{\circ} \mathrm{C}$.

\section{Measurement of flag leaf starch content}

Wheat flag leaf starch content according to the method described by Wang et al. (1993) with minor modifications. For all time points, leaf samples from each DH line and the parents were cut into small pieces. Leaf samples $(200 \mathrm{mg})$ were homogenized and placed in 50-mL centrifuge tubes, to which $15 \mathrm{~mL} \mathrm{80 \%} \mathrm{(v/v)} \mathrm{ethanol} \mathrm{was} \mathrm{added.} \mathrm{Samples} \mathrm{were}$ centrifuged at $10,000 \times g$ for $10 \mathrm{~min}$, after which the supernatant was discarded. The pelleted leaf material was stirred in $15 \mathrm{~mL} 80 \%$ (v/v) ethanol and used 80 water bath heating one hour, later cooled at room temperature for $30 \mathrm{~min}$. Samples were centrifuged at $10,000 \times g$ for $10 \mathrm{~min}$, after which the supernatant was collected in a beaker. This washing was repeated twice more (i.e. four washings) or until a test with anthrone reagent produced a negative result. The remaining residue following the extraction of sugar was resuspended in $10 \mathrm{~mL} \mathrm{35 \%} \mathrm{(v/v)} \mathrm{HClO}_{4}$, and starch was extracted overnight. Samples were used 80 water bath heating $10 \mathrm{~min}$ and centrifuged at $10,000 \times \mathrm{g}$ for $10 \mathrm{~min}$, after which the supernatant was collected and diluted with buffer solution to $25 \mathrm{ml}$. The resulting free glucose was analysed with an anthrone- $\mathrm{H}_{2} \mathrm{SO}_{4}$ reagent by Wang et al. (1993) using a UV$240 \mathrm{UV} / \mathrm{V}$ spectrophotometer (Shimadzu, Tokyo, Japan).

\section{Data and QTL analyses}

Mean, standard deviation, minimum, maximum, skewness, kurtosis, and coefficient variation were carried out using SPSS version 17.0 (SPSS, Chicago, IL, USA). Analysis of 
variance determined using SAS PROC MEANS. The genetic linkage map consisted of 368 markers were SSR loci, EST loci, inter-simple sequence repeat locus, and HMW-GS locus. The map covered a total length of $3074.1 \mathrm{cM}$, with an average distance of $8.35 \mathrm{cM}$ between adjacent markers. These linked markers formed 24 linkage groups over 21 chromosomes (Zhang et al. 2008; Zhao et al. 2010; Li et al. 2012). Genomic locations of marker loci/linkage groups were determined based on the wheat consensus map constructed by Somers et al. (2004). In our case, 30 loci $(7.03 \%)$ were found to map to different chromosomal locations than those on the Somers et al. (2004) map. Based on the recommendation of Doerge (2002), we used a maximum map distance of $10 \mathrm{cM}$ for genome-wide QTL scanning.

The genetic map was constructed with MAPMAKER/Exp version 3.0b (Lincoln et al. 1993). The commands "group" with LOD 4.0, "try", "compare", and "ripple" were used to develop the linkage map. Unlinked groups were oriented and placed for the same chromosome based on the microsatellite consensus map (Somers et al. 2004). The Kosambi mapping function (Kosambi 1944) was used to convert recombination fractions into cM as map distances. The linkage map was finally drawn using the software Mapchart, version 2.1 (Voorrips 2002).

Both unconditional and conditional QTLs were detected under a mixed linear model using IciMapping v3.2 (http://www.isbreeding.net/). Composite interval analysis was performed by forward-backward stepwise multiple linear regression, with a probability into and out of the model of 0.05 and a window size of $10 \mathrm{cM}$. For each data set, significant thresholds for QTL detection were calculated using 1,000 permutations and genomewide error rates of 0.10 (suggestive) and 0.05 (significant). The LOD score of 2.5 was chosen.

For QTL mapping, unconditional and conditional phenotypic values were arranged in the same data file. Unconditional phenotypic values corresponded to the data measured at different stages. Conditional phenotypic values at time $t$ given phenotypic values at time $t-1$ were predicted by the software program QGA Station v1.0 (http://ibi.zju.edu.cn/ software/qga/). Unconditional QTLs represent the cumulative effects of QTLs from the initial time to time $t$, while conditional QTLs correspond to the cumulative effects of QTLs from time $t-1$ to time $t$. QTLs were designated using the format "QTL + trait + chromosome" in accordance with international nomenclatural recommendations for QTLs in wheat and related species (McIntosh et al. 1994).

\section{Results}

\section{Phenotypic variations}

The results analysis of variance of wheat flag leaf starch contents over 2 years were listed in Table S1*. For FLSC, significant effects at 0.01 level were found for all variation sources except for Years $\times$ Lines $\times$ Sample times, Locations $\times$ Lines $\times$ Sample times and Lines $\times$ Sample times. Details regarding the FLSC of the DH population and the parents

*Further details about the Electronic Supplementary Material (ESM) can be found at the end of the article. 
in six environments at six time points are provided in Table S2. Strong transgressive segregations were observed, indicating that alleles with positive effects were distributed among the parents. The absolute values of the skewness and kurtosis for the FLSC distributions in the DH population were less than 1. This suggested that the trait approximately followed normal distributions, and the experimental data were suitable for QTL analyses. The FLSC increased from 7:00 to 19:00 and then decreased from 19:00 to the following 7:00 (Wang et al. 2014).

\section{Unconditional quantitative trait loci detected for the diurnal wheat flag leaf starch content}

Eight unconditional QTLs were identified for the FLSC at the six sampling stages in six environments (Table S3; Fig. S1). Unconditional QTLs with a contribution rate of 3.1$15.8 \%$ for the FLSC were located on six chromosomes (i.e. 1B, 1D, 3A, 4A, 7B, and 7D). An independent analysis of the data generated for different environments identified four main additive QTLs (i.e. $q F L S-1 B, q F L S-1 D-1, q F L S-4 A$, and $q F L S-7 D-1$ ) in two of six environments. Two QTLs ( $q F L S-1 D-1$ and $q F L S-7 D-1)$ were steadily expressed at the same stages in different environments, but had different effects. The other additive QTLs were detected in only one environment.

Additional details were revealed when the data for six environments were analysed together. No QTL was detected at all six stages. The $q F L S-1 D-2$ was the only QTL identified at three stages (i.e. $\mathrm{T}_{2}, \mathrm{~T}_{4}$, and $\mathrm{T}_{5}$ ), and explained $12.7 \%, 8.0 \%$, and $15.8 \%$ of the total phenotypic variance, respectively. Three QTLs (i.e. $q F L S-1 B, q F L S-4 A$, and $q F L S$ $7 D-1$ ) were detected at two stages, and explained $17.7 \%, 15.3 \%$, and $26.0 \%$ of the total phenotypic variance, respectively. Additionally, $q F L S-7 D-2$, which was detected at stage $\mathrm{T}_{4}$, explained $10.2 \%$ of the total variance. The remaining QTL was identified at one stage. On chromosome 7, QTLs detected at stages $\mathrm{T}_{1}, \mathrm{~T}_{2}$, and $\mathrm{T}_{4}$ explained $36.2 \%$ of the phenotypic variance.

Several unconditional QTLs affecting the diurnal wheat FLSC were detected at different developmental stages. According to the conditional QTL mapping results, seven additional QTLs detected at the $\mathrm{T}_{4}$ stage explained $76.8 \%$ of the phenotypic variance. The results of the unconditional QTL mapping suggested that more QTLs could be detected using phenotypic data collected at different time points throughout the plant developmental stages than with data from only one stage. The detection of different QTLs at various time points indicated that the expression of the genes regulating the wheat FLSC may change over the course of a day.

\section{Conditional quantitative trait loci detected for the diurnal wheat flag leaf starch content}

The nine conditional QTLs for the FLSC detected at six stages were distributed on eight chromosomes (Fig. S1; Table S4). These QTLs explained 6.8-22.5\% of the phenotypic variation. The conditional QTL $q F L S-3 A-1$ was identified in two environments, and ex- 
plained $11.2 \%$ and $9.5 \%$ of the total phenotypic variance in these environments. The QTL ( $q F L S-7 D-1)$ was detected at four stages, and explained 15.3\%, 14.4\%, 12.7\%, and 12.8\% of the total phenotypic variance at these stages. Additionally, $q F L S-7 D-2$, which was detected at two stages, explained $10.1 \%$ and $7.8 \%$ of the total phenotypic variance. The QTL ( $q F L S-7 D-2)$ was detected at one stage, and explained $22.5 \%$ of the phenotypic variance. Four and three QTLs were identified at the $\mathrm{T}_{3} \mid \mathrm{T}_{2}$ and $\mathrm{T}_{4} \mid \mathrm{T}_{3}$ stages, and accounted for $34.1 \%$ and $45.8 \%$ of the total phenotypic variance, respectively.

We mapped 12 QTLs, and a comparison of the unconditional and conditional QTL mapping results (Tables S3 and S4; Fig. S1) revealed nine unconditional QTLs and eleven conditional QTLs. Eight QTLs were both unconditional and conditional QTLs. Of these, four unconditional QTLs (i.e. $q F L S-1 B, q F L S-1 D-1, q F L S-4 A$, and $q F L S-7 D-1$ ) and one conditional QTL (i.e. $q F L S-3 A-1$ ) were identified in two of six environments. Based on the unconditional QTL mapping results, $q F L S-1 D-2$ and $q F L S-7 D-1$ having the largest contribution to the phenotypic variation (more than $10 \%$ ) and were detected more than one stage. In contrast, three QTLs (i.e. $q F L S-1 A, q F L S-3 A-1$, and $q F L S-7 D-1$ ) were detected using the conditional QTL mapping method. Our findings indicated that combining unconditional and conditional QTL mapping methods enabled the detection of gene expression changes associated with quantitative traits.

\section{Discussion}

\section{Temporal expression of quantitative trait loci}

Several QTLs affecting the diurnal FLSC have been detected by QTL mapping (Calenge et al. 2006; Ishimaru et al. 2005, 2007). Previous studies on these QTLs focused on a single developmental stage (i.e. usually the harvest stage). Thus, they did not reveal changes to QTL expression during trait development. To clarify the mechanism regulating the accumulation of starch in leaves, we used unconditional and conditional QTL mapping methods to investigate the developmental changes in the wheat diurnal FLSC during the early grain-filling stage in different environments.

We identified eight unconditional additive QTLs and nine conditional additive QTLs for the FLSC during the early grain-filling stage over 2 years at three locations (Table S3; Fig. S1). The number of QTLs related to the wheat FLSC and their genetic effects differed among various stages. One unconditional QTL (i.e. $q F L S-1 D-2$ ) was identified at three stages (i.e. $\mathrm{T}_{2}, \mathrm{~T}_{4}$, and $\mathrm{T}_{5}$ ), while another three unconditional QTLs (i.e. $q F L S-1 B$, $q F L S-4 A$, and $q F L S-7 D-1)$ were detected at two stages. The conditional QTL $q F L S-7 D-1$ was identified at four stages, while $q F L S-7 D-2$ was detected at two stages. These QTLs reflected the continuous expression of specific genes, and significantly affected the FLSC. They are more important than the remaining QTLs that were detected at only one stage because the FLSC increases over a long period during different developmental stages. The pyramiding of positive alleles (expressed at consecutive stages) from different parents by marker-assisted selection may lead to increased FLSC, thereby improving wheat seed yields. 
Most QTLs were detected at only one stage, and no QTL was detected at all five analysed periods. These results indicate that the expression levels of the genes controlling the FLSC exhibit dynamic changes during plant development. Most of the genes associated with the identified QTLs are only briefly expressed, although some are expressed over a longer period under very specific situations. However, none of the genes are expressed throughout the day and night. Our results are clearly in agreement with the developmental genetics principle that states genes are selectively expressed at different growth stages (Atchley and Zhu 1997). These results may improve our understanding of the genetic control of the diurnal FLSC.

\section{Combining conditional and unconditional quantitative trait loci}

Unconditional QTL mapping can be used to determine the results of accumulated genetic effects at measurement time $t$, thus providing information about "static" gene expression at time $t$. In contrast, conditional QTL mapping can reveal the gene expression levels from time $t-1$ to time $t$, thereby reflecting the net effects of gene expression over a specific period and providing information about "dynamic" gene expression at time $t$ (Bian et al. 2015; Shang et al. 2016; Zhang et al. 2016). Combining unconditional and conditional QTL mapping results enables the detection of multiple QTLs, helps verify the results of the two methods, and simultaneously provides static and dynamic gene expression details (Jiang et al. 2013).

In this study, four additive QTLs (i.e. $q F L S-1 A, q F L S-2 D, q F L S-3 A-1$, and $q F L S-5 A$ ) were detected using a conditional QTL mapping method, but not with an unconditional QTL mapping strategy. Genes with very small effects during a specific growth period may be undetectable using the unconditional QTL mapping approach. In contrast, three additive QTLs (i.e. $q F L S-1 B, q F L S$ - $1 D$-2, and $q F L S$ - $3 A$-2) were identified using an unconditional QTL mapping method, but not with a conditional QTL mapping procedure. Genes with opposing effects may be expressed at the same or nearby locations during previous growth periods. Consequently, variations in the cumulative gene effects may be diminished to the point of being undetectable. Therefore, a combination of conditional and unconditional QTL mapping methods that considers genetic effects may lead to a deeper characterization of the complexity of QTLs associated with the development of quantitative traits. In other words, dynamic mapping of QTLs based on conventional and conditional methods represents a powerful approach to revealing the genetic basis underlying the development of quantitative traits.

The number of unconditional QTLs differed among the analysed stages. We identified one, three, one, eight, and one unconditional QTLs at $\mathrm{T}_{1}, \mathrm{~T}_{2}, \mathrm{~T}_{3}, \mathrm{~T}_{4}$, and $\mathrm{T}_{5}$, respectively. There were no unconditional QTLs detected at $\mathrm{T}_{6}$. The greater number of QTLs at $\mathrm{T}_{4}$ (i.e. 19:00) is consistent with the fact that the transient starch in flag leaves starts to degrade during the transition from day to night. Additionally, $\mathrm{T}_{6}$ (i.e. 3:00) corresponds to the middle of the night when most of the flag leaf starch content has degraded, which is consistent with a lack of detected unconditional QTLs at this time point. 


\section{Comparisons with previous studies}

Molecular mapping experiments have revealed that several QTLs regulate starch contents in Arabidopsis thaliana and rice. There are no reports describing the analysis of QTLs for wheat flag leaf starch content (FLSC). But some QTLs controlling grain or flour starch content on 1A, 1B, 1D, 2A, 2D, 3A, 4A, 5B, 7A, 7B, and 7D chromosomes were found using different mapping populations (McCartney et al. 2006; Sun et al. 2008; Deng et al. 2015). Compared with previous reports, we detected QTLs for starch content on 1B, 1D, 2D, 3A, 4A, 5A, 7B, and 7D chromosomes by unconditional and conditional QTL mapping. By comparing these chromosomes, several chromosomes were same for starch content. There were some important critical loci controlling starch synthesis on 1D, 4A, 7B, and 7D chromosomes detected in the present research.

In summary, the dynamic QTLs identified in this study facilitate better understanding of the genetic regulation of FLSC. However, the final aim of the QTL mapping was to effectively utilize the detected QTLs. Any identified QTLs that are highly stable (i.e. environmentally stable) and that explain more than $10 \%$ of the phenotypic variation may be candidates for MAS in wheat breeding programs. We identified 6 conditional and 4 unconditional relatively stable QTLs for protein fraction content that explained more than $10 \%$ of the phenotypic variation. Five QTLs ( $q F L S-1 B, q F L S-1 D-1, q F L S-3 A-1, q F L S$ $4 A$ and $q F L S-7 D-1)$ were expressed in several environments. We also identified 3 QTLs ( $q F L S-1 D-2, q F L S-7 D-1$ and $q F L S-7 D-2)$ that were expressed in consecutive stages. It should be noted, however, that relatively stable QTLs with small effects should be chosen carefully for marker-assisted selection in breeding programs (Jiang et al. 2010). QTLs for two or more important traits in non-homologous regions could facilitate pyramiding of favourable alleles by MAS for simultaneous improvement of those traits. To reduce the negative effect of unfavourable correlations among alleles controlling different traits, larger segregating populations must be developed for fine QTL mapping and further MAS in breeding programs. Exploitation of additional markers (new SSRs and SNPs) for mapping the target regions is therefore needed.

\section{Acknowledgements}

This study was supported by the Hebei Basic Key Research Program (17966314D) and the Fund for Team Building of Crop Science Hebei Agricultural University (TD2016C3081).

\section{References}

Atchley, W.R., Zhu, J. 1997. Developmental quantitative genetics, conditional epigenetic variability and growth in mice. Genetics 147:765-776.

Bian, Y.L., Gu, X., Sun, D.L., Wang, Y.J., Yin, Z.T., Deng, D.X., Wang, Y.Q., Li, G.S. 2015. Mapping dynamic QTL of stalk sugar content at different growth stages in maize. Euphytica 205:85-94.

Calenge, F., Saliba-Colombani, V., Mahieu, S., Loudet, O., Daniel-Vedele, F., Krapp, A. 2006. Natural variation for carbohydrate content in Arabidopsis. Interaction with complex traits dissected by quantitative genetics. Plant Physiol. 141:1630-1643. 
Deng, Z.H., Hu, S.N., Chen, F., Li, W.J., Chen, J.S., Sun, C.S., Zhang, Y.X., Wang, S.Y., Song, X.J. Tian, J.C. 2015. Genetic dissection of interaction between wheat protein and starch using three mapping populations. Mol. Breed. 35:1-9.

Doerge, R.W. 2002. Mapping and analysis of quantitative trait loci in experimental populations. Nat. Rev. Genet. 3:43-52.

Fondy, B.R., Geiger, D.R. 1985. Diurnal changes in allocation of newly fixed carbon in exporting sugar beet leaves. Plant Physiol. 78:753-757.

Fondy, B.R., Geiger, D.R., Servaites, J.C. 1989. Photosynthesis, carbohydrate metabolism, and export in Beta vulgaris L. and Phaseolus vulgaris L. during square and sinusoidal light regimes. Plant Physiol. 89:396402.

Guo, C.Q., Bai, Z.A., Liao, P.A., Jin, W.K. 2004. New high quality and yield wheat variety Yumai 57. Crop Sci. 4:54.

Hai, Y., Kang, M.H. 2007. Breeding of a new wheat variety Huapei 3 with high yield and early maturity. J. Henan Agric. Sci. 5:36-37.

Ishimaru, K., Kashiwagi, T.N., Hirotsu, N., Madoka, Y. 2005. Identification and physiological analyses of a locus for rice yield potential across the genetic background. J. Exp. Bot. 5:2745-2753.

Ishimaru, K., Hirotsu, N., Madoka, Y., Kashiwagi, T.N. 2007. Quantitative trait loci for sucrose, starch, and hexose accumulation before heading in rice. Plant Physiol. Biochem. 45:799-804.

Jenner, C.F., Ugalde, T.D., Aspinall, D. 1991. The physiology of starch and protein deposition in the endosperm of wheat. J. Plant Physiol. 18:211-226.

Jiang, P., Wan, Z.Y., Wang, Z.X., Li, S.S., Sun, Q.Q. 2013. Dynamic QTL analysis for activity of antioxidant enzymes and malondialdehyde content in wheat seed during germination. Euphytica 190:75-85.

Jiang, Z.F., Han, Y.P., Teng, W.L., Zhang, Z.C., Sun, D.S., Li, Y.H., Li, W.B. 2010. Identification of QTL underlying the filling rate of protein at different developmental stages of soybean seed. Euphytica 175:227-236.

Kato, K., Miura, H., Sawada, S. 2000. Mapping QTLs controlling grain yield and its components on chromosome 5A of wheat. Theor. Appl. Genet. 101:1114-1121.

Kosambi, D.D. 1944. The estimation of map distances from recombination values. Ann. Eugen. 12:172-175.

Li, Q.F., Zhang, Y., Liu, T.T., Wang, F.F., Liu, K., Chen, J.S., Tian, J.C. 2015. Genetic analysis of kernel weight and kernel size in wheat (Triticum aestivum L.) using unconditional and conditional QTL mapping. Mol. Breed. 35:1-15.

Li, W.F., Liu, B., Peng, T., Yuan, Q.Q., Han, S.X., Tian, J.C. 2012. Detection of QTL for kernel weight, grain size, and grain hardness in wheat using DH and immortalized F2 population. Sci. Agric. Sin. 45:3453-3462.

Lincoln, S.E., Daly, M.J., Lander, E.S. 1993. Constructing Genetic Maps with MAPMAKER/EXP Version 3.0: A Tutorial and Reference Manual. White Head Inst. Biomed. Res. Tech. Rpt, 3rd edn. White Head Institute for Biomedical Research. Cambridge, UK. 97 pp.

Matsushima, S. 1966. Theory of yield determination and its application. Crop Sci. Rice 365:12-13.

McCartney, C.A., Somers, D.J., Lukow, O., Ames, N., Noll, J., Cloutier, S., Humphreys, D.G., Mccallum, B.D. 2006. QTL analysis of quality in traits in the spring wheat cross RL $4452 \times \mathrm{AC}$ Domain. Plant Breed. 125:565-575.

McIntosh, R.A., Hart, G.E., Gale, M.D. 1994. Catalogue of gene symbols for wheat. Wheat Inf. Serv. 79:47-56.

Shang, L.G., Wang, Y.M., Cai, S.H., Ma, L.L., Liu, F., Chen, Z.W., Su, Y., Wang, K.B., Hua, J.P. 2016. Genetic analysis of upland cotton dynamic heterosis for boll number per plant at multiple developmental stages. SCI REP-UK 6:35515.

Smith, A.M., Zeeman, S.C., Thorneycroft, D., Smith, S.M. 2003. Starch mobilization in leaves. J. Exp. Bot. 54:577-583.

Somers, D.J., Isaac, P., Edwards, K. 2004. A high-density micro-satellite consensus map for bread wheat (Triticum aestivum L.). Theor. Appl. Genet. 109:1105-1114.

Sun, H.Y., Lu, J.H., Fan, Y.D., Zhao, Y., Kong, F., Li, R.J., Wang, H.G., Li, S.S. 2008. Quantitative trait loci (QTLs) for quality traits related to protein and starch in wheat. Prog. Nat. Sci. 18:825-831.

Voorrips, R.E. 2002. MapChart: software for the graphical presentation of linkage maps and QTLs. J. Hered. 93:77-78. 
Wang, D.L., Zhu, J., Li, Z.K.L., Paterson, A.H. 1999. Mapping QTLs with epistatic effects and QTL $\times$ environment interactions by mixed linear model approaches. Theor. Appl. Genet. 99:1255-1264.

Wang, F., Sanz, A., Brenner, M.L., Smith, A. 1993. Sucrose synthase, starch accumulation, and tomato fruit sink strength. Plant Physiol. 101:321-327.

Wang, G., Zhao, Y., Yang, X.F., Li, X.Y., Yang, X.J. 2014. The circadian rhythm of flag leaf starch accumulation in wheat. J. Hebei Agric. Univ. 37:1-5.

Weise, S.E., Weber, A.P.M., Sharkey, T.D. 2004. Maltose is the major form of carbon exported from the chloroplast at night. Planta 218:474-482.

Xu, H.Y., Zhao, J.S. 1995. Canopy photosynthesis capacity and the contribution from different organs in high yielding winter wheat. Sci. Agric. Sin. 21:204-209.

Xu, Y.F., Li, S.S., Li, L.H., Ma, F.F., Fu, X.Y., Shi, Z.L., Xu, H.X., Ma, T.P., An, D.G. 2017. QTL mapping for yield and photosynthetic related traits under different water regimes in wheat. Mol. Breed. 37:1-18.

Zeeman, S.C., Smith, S.M., Smith, A.M. 2004. The breakdown of starch in leaves. New Phytol. 163:247-261.

Zeeman, S.C., Smith, S.M., Smith, A.M. 2007. The diurnal metabolism of leaf starch. Biochem. J. 401:13-28.

Zhang, H., Wang, H.G. 2015. QTL mapping for traits related to P-deficient tolerance using three related RIL populations in wheat. Euphytica 203:505-520.

Zhang, K.P., Zhao, L., Tian, J.C., Chen, G.F., Jiang, X.L., Liu, B. 2008. A genetic map constructed using a doubled haploid population derived from two elite Chinese common wheat varieties. J. Int. Plant Bio. 50:941-950.

Zhang, Z.H., Wu, X.Y., Shi, C.N., Wang, R.N., Li, S.F., Wang, Z.H., Liu, Z.H., Xue, Y.D., Tang, G.L., Tang, J.H. 2016. Genetic dissection of the maize kernel development process via conditional QTL mapping for three developing kernel-related traits in an immortalized F2 population. Mol. Genet. Genomics 291:437457.

Zhao, L., Zhang, K.P., Liu, B., Deng, Z.Y., Qu, H.L., Tian, J.C. 2010. A comparison of grain protein content QTLs and flour protein content QTLs across environments in cultivated wheat. Euphytica 174:325-335.

Zhu, J. 1995. Analysis of conditional genetic effects and variance components in developmental genetics. Genetics 141:1633-1639.

\section{Electronic Supplementary Material (ESM)}

Electronic Supplementary Material (ESM) associated with this article can be found at the website of CRC at http://www.akademiai.com/content/120427/

Electronic Supplementary Table S1. Analysis of variance of wheat flag leaf starch contents

Electronic Supplementary Table S2. Statistical analysis of flag leaf starch content in parents Huapei 3 (HP3) and Yumai 57 (YM57) and the doubled-haploid (DH) population at different stages for 2 years in three locations

Electronic Supplementary Table S3. Additive effects of quantitative trait loci (QTLs) for the wheat flag leaf starch content based on an unconditional QTL mapping method

Electronic Supplementary Table S4. Additive effects of quantitative trait loci (QTLs) for the wheat flag leaf starch content based on a conditional QTL mapping method

Electronic Supplementary Figure S1. Chromosomal locations of quantitative trait loci (QTLs) associated with the flag leaf starch content 